History, Philosophy \& Theory of the Life Sciences

Sebastian Normandin Charles T. Wolfe Editors

\title{
Vitalism and the
} Scientific Image in Post-Enlightenment Life Science, $1800-2010$ 